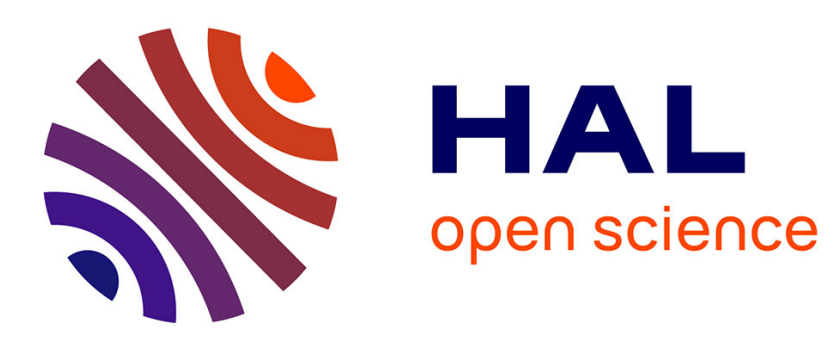

\title{
Étude théorique et expérimentale des trajectoires ioniques transgaussiennes d'une lentille magnétique blindée à symétrie de révolution
}

Michel Del'Homme

\section{- To cite this version:}

Michel Del'Homme. Étude théorique et expérimentale des trajectoires ioniques transgaussiennes d'une lentille magnétique blindée à symétrie de révolution. Revue de Physique Appliquée, 1969, 4 (4), pp.495-500. 10.1051/rphysap:0196900404049500 . jpa-00243318

HAL Id: jpa-00243318

https://hal.science/jpa-00243318

Submitted on 1 Jan 1969

HAL is a multi-disciplinary open access archive for the deposit and dissemination of scientific research documents, whether they are published or not. The documents may come from teaching and research institutions in France or abroad, or from public or private research centers.
L'archive ouverte pluridisciplinaire HAL, est destinée au dépôt et à la diffusion de documents scientifiques de niveau recherche, publiés ou non, émanant des établissements d'enseignement et de recherche français ou étrangers, des laboratoires publics ou privés. 


\title{
ÉTUDE THÉORIQUE ET EXPÉRIMENTALE DES TRAJEGTOIRES IONIQUES TRANSGAUSSIENNES D'UNE LENTILLE MAGNÉTIQUE BLINDÉE A SYMÉTRIE DE RÉVOLUTION
}

\author{
Par Michel DEL'HOMME, \\ Faculté des Sciences, Laboratoire de Spectrométrie de Masse, avenue du Recteur-Pineau, 86-Poitiers. \\ (Reçu le 16 juillet 1969.)
}

\begin{abstract}
Résumé. - Un procédé de calcul de trajectoires transgaussiennes de particules chargées dans une lentille magnétique blindée a été proposé dans une précédente publication [1], ce calcul étant établi, en fait, à partir de mesures de flux magnétique, et non de champ.

Ce procédé est appliqué à des ions $\mathrm{He}^{+}$traversant une telle lentille. Le dispositif expérimental d'investigation du flux magnétique est décrit, ainsi que le processus d'exploitation des résultats, permettant la détermination sur ordinateur des trajectoires ioniques. Un banc d'optique ionique utilisant cette lentille permet de vérifier que les trajectoires calculées et expérimentales sont très voisines, l'écart entre les distances focales théoriques et réelles restant inférieur à $1,5 \%$.
\end{abstract}

Abstract. - A method for the determination of transgaussian trajectories of charged particles in a shielded magnetic lens has been proposed in a previous paper [1] ; this calculation is obtained, in fact, from magnetic flux measurements and not from field measurements.

In the present paper, this method is applied for $\mathrm{He}^{+}$ions. The apparatus of investigation of the magnetic flux is described, and also the process using these experimental results, for the calculation of the trajectories. An ion optical bench using this lens, made us able to verify that experimental and computed trajectories are in a good agreement, the difference between computed and measured focal lengths being below $1.5 \%$.

I. Introduction. - L'étude du mouvement d'une particule chargée, dans un champ magnétique à symétrie de révolution, nécessite la connaissance de la distribution du potentiel-vecteur A dont dérive le champ. $\mathrm{Si}$, pour les trajectoires paraxiales, le problème se résout facilement à partir de mesures du champ magnétique sur l'axe, il n'en est plus de même pour les trajectoires marginales, le champ magnétique au voisinage de l'entrefer des lentilles utilisées étant très supérieur à ce qu'il est sur l'axe. Un tel mode d'utilisation d'une lentille magnétique de révolution se rencontre en spectrométrie $\beta$ ou dans certains spectromètres de masse (utilisés plutôt comme détecteurs de fuite) : dans les deux cas, la séparation des particules suivant leur quantité de mouvement est assurée par l'utilisation exclusive des rayons marginaux. D'autre part, la connaissance précise de ces trajectoires marginales permettra, le cas échéant, le calcul des aberrations d'ouverture et de chromatisme. Dans une récente publication [1], nous avons présenté une méthode de détermination de ces trajectoires transgaussiennes et nous nous proposons, dans cette étude, de l'appliquer à la détermination théorique de trajectoires ioniques à l'intérieur d'une lentille magnétique blindée.
II. Rappel du principe. - Considérons une boucle circulaire de rayon $r$ axée sur l'axe de révolution $O z$; le flux $\Phi(r, z)$ produit à l'intérieur de cette boucle par le champ $H$, et le potentiel vecteur $A(r, z)$ sont liés par la relation :

$$
A(r, z)=\frac{\Phi(r, z)}{2 \pi r} .
$$

L'équation rigoureuse dans le plan méridien mobile de la trajectoire d'une particule de charge $e$ et de masse $m$, issue d'un point de l'axe, et ayant subi l'action d'une tension accélératrice $V_{0}$, peut se mettre sous la forme :

$$
r^{\prime \prime}=\frac{1+r^{\prime 2}}{2 \chi}\left(\frac{\partial \chi}{\partial r}-r^{\prime} \frac{\partial \chi}{\partial z}\right)
$$

$\chi$ étant une fonction de $\Phi(r, z)$ par l'intermédiaire de la relation (3) suivante :

$$
\chi(r, z)=V_{0}-\frac{e}{8 m \pi^{2} r^{2}} \Phi^{2}(r, z) .
$$

L'équation (2) est identique dans la forme à celle décrivant la trajectoire d'une particule chargée dans un champ électrique à symétrie axiale, la fonc- 
tion $\chi(r, z)$ remplaçant la fonction potentiel électrique $V(r, z)$; la connaissance, par l'intermédiaire de $\Phi(r, z)$ de la distribution $\chi(r, z)$ dans le plan $(O r$, $O z)$, permet donc le calcul des trajectoires par les procédés classiques utilisés pour les lentilles électriques, l'avantage de cette méthode étant d'éviter toute intégration numérique pour l'obtention du potentielvecteur et de faire intervenir dans l'équation (2) une grandeur directement accessible à l'expérience.

III. Détermination expérimentale de la variable $\chi(r, z) .-1$. Processus De Mesure. - Il s'agit de déterminer $\chi(r, z)$ à l'intérieur d'une lentille magnétique blindée. Cette détermination se ramène selon l'équation (3) à la mesure de $\Phi(r, z)$. Celle-ci peut se faire simplement en utilisant une bobine de mesure de rayon $r$ axée sur $O z$ au point d'abscisse $z$, et reliée à un galvanomètre balistique. On mesure la déviation galvanométrique produite par l'inversion brusque du courant magnétisant dans le circuit de la lentille magnétique. Naturellement, le galvanomètre doit être préalablement étalonné à l'aide d'un champ uniforme connu. Pour déterminer $\Phi\left(r, z_{0}\right)$, il suffira de centrer au point $z_{0}$ une bobine de rayon $r$; si $r$ est la variable, on utilisera un jeu de bobines de diamètres différents. Pour les valeurs suffisamment grandes de $r$ (ce qui est le cas considéré ici), une seule spire peut être suffisante, la surface de la bobine étant élevée. Pour les valeurs de $r$ se rapprochant de l'axe, la sensibilité diminue proportionnellement à $r^{2}$, mais on peut l'améliorer en augmentant le nombre de spires, de façon limitée toutefois. Le nombre très faible des spires permettra d'obtenir une excellente précision sur la définition de $r$ et de $z$ en même temps qu'une réalisation simple et rapide. La précision relative des mesures du galvanomètre doit croître d'autre part avec la valeur de $r$, par suite de l'augmentation de $r$ et de $H$, d'où une précision particulièrement bonne au voisinage de l'entrefer de la lentille.

2. Lentille magnétique et Dispositif DE MEsUre. - a) Lentille magnétique. - Nous avons étudié par la méthode ci-dessus la répartition de la fonction $\chi$ à l'intérieur d'une lentille blindée de révolution ( $f g .6$ ) de 2900 spires et de $26 \mathrm{~mm}$ de diamètre interne. Cette lentille est entièrement entourée d'un blindage en fer Armco dans lequel est ménagé un entrefer de $6 \mathrm{~mm}$. Une alimentation «Sorensen QR B 40-2 » fournit un courant magnétisant régulé en intensité.

L'origine des axes $O z$ et $O r$ a été prise au centre de l'entrefer sur l'axe de révolution de la lentille. Les mesures ont été effectuées à l'intérieur d'un domaine rectangulaire ( fig. 1) délimité par les valeurs suivantes : $r$, variable de 7 à $11 \mathrm{~mm}$; $z$, variable de $-20 \mathrm{~mm}$ à $+20 \mathrm{~mm}$ de chaque côté de l'entrefer, le champ magnétique pouvant être considéré comme négligeable à l'extérieur de ces abscisses limites.

b) Bobines de mesure, bobine de référence (fig. 2). Chaque bobine de mesure b comporte 5 spires juxta-

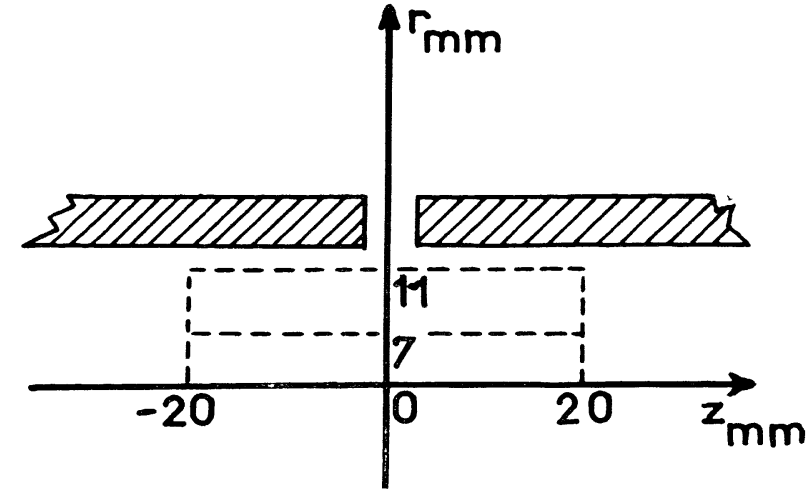

FIG. 1. - Domaine de mesure de $\chi$ dans la lentille.

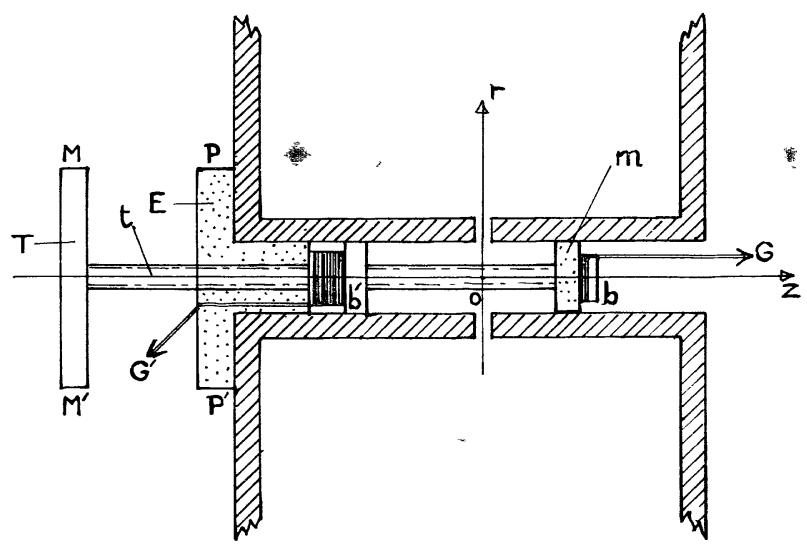

Frg. 2. - Dispositif de mesure du flux.

posées de fil de cuivre émaillé ( $\varnothing 0,05 \mathrm{~mm}$ ) enroulé sur un mandrin $\mathrm{m}$ en plexiglas, coulissant à frottement doux dans le canal de la lentille. La bobine de mesure $b$, reliée à un galvanomètre $G$, est solidaire d'une tige filetée $t$, manœuvrée par un tambour $T$, et se déplaçant dans la monture $\mathrm{E}$; chaque rotation d'un tour du tambour $\mathrm{T}$ permet ainsi un déplacement de la bobine $\mathrm{b}$, de $1 \mathrm{~mm}$ le long de l'axe $O z$. La position de départ de cette bobine b sur l'axe $O z$ est mesurée avec précision au palmer entre les flasques $\mathrm{MM}^{\prime}$ et $\mathrm{PP}^{\prime}$, compte tenu de la largeur $(0,25 \mathrm{~mm})$ de l'enroulement; d'autre part, il est important de s'assurer de la reproductibilité de la valeur et des conditions d'établissement du courant dans la lentille, lors d'une série de mesures ou lors du changement de bobine exploratrice. Ceci est réalisé par une deuxième bobine $\mathrm{b}^{\prime}$ solidaire $\mathrm{du}$ flasque $\mathrm{PP}^{\prime}$ et reliée à un deuxième galvanomètre $\mathrm{G}^{\prime}$; on mesure le flux produit par le champ de fuite de la lentille dans cette bobine $\mathrm{b}^{\prime}$; celui-ci étant très faible, on enroule un grand nombre de spires de grand diamètre, de manière à obtenir une déviation galvanométrique suffisante (2000 spires sur un diamètre interne de $20 \mathrm{~mm}$ dans notre cas). Cette bobine étant fixe à l'intérieur de la lentille, le maintien de la grandeur de la déviation 
du galvanomètre $\mathrm{G}^{\prime}$ nous assure de la bonne reproductibilité des conditions d'établissement et de la valeur du courant magnétisant, une correction étant apportée le cas échéant; l'ensemble du dispositif est réalisé en plexiglas afin de ne pas perturber les lignes de champ.

Une série de 9 bobines a été réalisée, dont les rayons varient de 7 à $11 \mathrm{~mm}$, de 0,5 en $0,5 \mathrm{~mm}$. Le diamètre de ces bobines ainsi que leur position le long de l'axe $\mathrm{Oz}$ sont donc parfaitement définis, les relevés étant faits de $\mathrm{mm}$ en $\mathrm{mm}$ à l'aide du dispositif de la figure 2 .

c) Étalonnage. - Ces mesures ont été effectuées pour un courant magnétisant dans la lentille, de 1 ampère, donnant un champ d'environ 1500 gauss à l'origine $O$. L'étalonnage du galvanomètre $\mathrm{G}$ a été réalisé à l'aide d'un champ uniforme de 225 gauss créé par un solénoïde auxiliaire, dans lequel l'une des bobines exploratrices $\mathrm{b}$ a été positionnée de façon invariable au centre du champ. Nous avons obtenu une correspondance de $0,523 \times 10^{-6}$ weber par $\mathrm{mm}$ de déviation. Cet étalonnage du galvanomètre nécessite quelques précautions; en effet, les conditions d'établissement du courant magnétisant dans la lentille blindée d'une part, et dans la bobine d'étalonnage délivrant le champ uniforme d'autre part, doivent être les mêmes. Dans notre cas, la self-inductance de la bobine d'étalonnage étant beaucoup plus faible que celle de la lentille blindée, ces conditions ne se trouvent donc pas réalisées. Pour éviter cet inconvénient, nous avons, lors de l'étalonnage, mis les deux bobinages en série afin d'obtenir des conditions d'établissement du courant magnétisant sensiblement identiques.

3. Distribution de $\chi, \frac{\partial \chi}{\partial r}, \frac{\partial \chi}{\partial z}$. - A partir des mesures de $\Phi(r, z)$, on détermine facilement $\chi(r, z)$ suivant l'équation (3); on en déduit ensuite les deux termes $\frac{\partial \chi}{\partial r}$ et $\frac{\partial \chi}{\partial z}$ nécessaires à la résolution de l'équation (2). La précision relative sur $\Phi(r, z)$, donc celle de la lecture de la déviation galvanométrique, est faible pour les positions éloignées de l'origine, donc de l'entrefer; mais dans ce cas le terme en $\Phi$ étant faible, $\chi(r, z)$ est peu différent de $V_{0}$, et la précision relative de $\chi(r, z)$ reste bonne. Quand on se rapproche de l'origine, le terme en $\Phi$ prend de plus en plus d'importance dans la valeur de $\chi(r, z)$, mais sa précision croît en même temps que la déviation galvanométrique. Il en résulte que la détermination totale de la distribution de $\chi(r, z)$ est faite avec une précision d'environ $1 \%$ en valeur relative.

Les courbes obtenues, pour un ion d'hélium et

FIG. 3 a, 3 b et 3 c. - Courbes donnant la distribution de $\chi, \frac{\partial \chi}{\partial z}, \frac{\partial \chi}{\partial r}$ en fonction de $r$ :

$$
\begin{aligned}
& \text { Pour } r_{1}=7 \mathrm{~mm} . \\
& ---- \text { Pour } r_{2}=9 \mathrm{~mm} . \\
& --- \text { Pour } r_{3}=11 \mathrm{~mm} .
\end{aligned}
$$

pour une tension accélératrice $V_{0}$ de 200 volts, à l'intérieur du domaine actif de la lentille sont celles de la figure 3 .
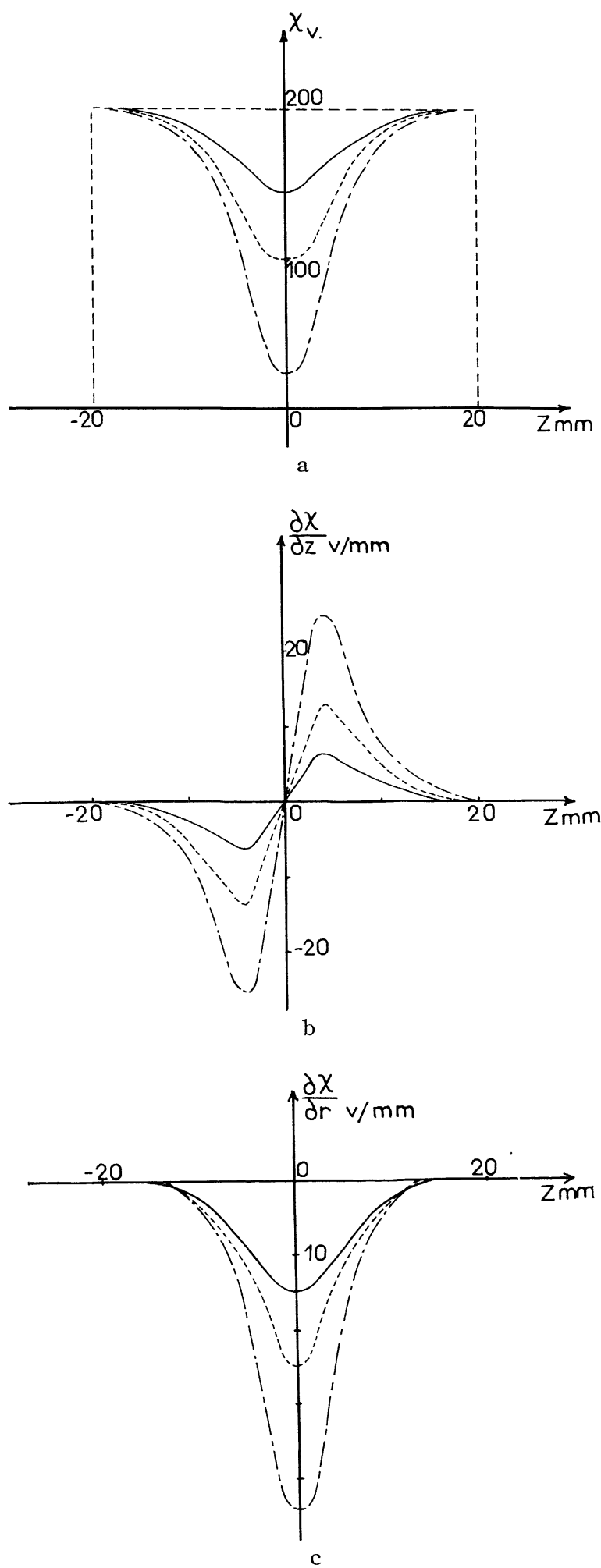
Afin de ne pas trop surcharger les graphiques, nous n'avons reproduit que celles relatives à trois valeurs de $r\left(r_{1}=7 \mathrm{~mm}, r_{2}=9 \mathrm{~mm}, r_{3}=11 \mathrm{~mm}\right)$ et pour 200 volts de tension accélératrice. Pour une autre valeur $V_{0} \pm \Delta V$ de cette tension, seul le terme $\chi$ (courbes de la figure 3 a) est affecté. Pour obtenir le réseau de courbes correspondant à cette nouvelle tension $V_{0} \pm \Delta V$, il suffit d'opérer une translation du réseau le long de l'axe $\chi$, d'amplitude $\pm \Delta V$.

IV. Calcul des trajectoires marginales. - a) CARACTÉRISTIQUES INITIALES. - Cette détermination de $\chi(r, z)$ a été appliquée au calcul théorique des trajectoires ioniques pour des ions d'hélium accélérés sous diverses tensions et traversant la lentille à différentes ordonnées dans le domaine transgaussien ( fig. 4).

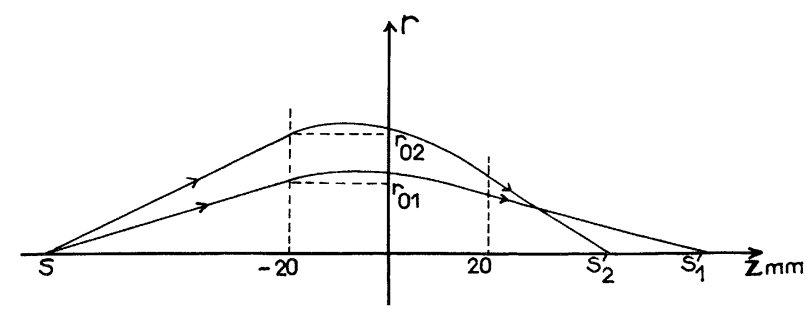

Fig. 4. - Allure des trajectoires suivant la valeur de $r_{0}\left(V_{0}=\right.$ Cte $)$.

Les calculs ont été effectués à l'aide d'un calculateur IBM 1620 nous fournissant pour les trajectoires, d'une part les ordonnées point par point, de $\mathrm{mm}$ en $\mathrm{mm}$ entre les abscisses $-20 \mathrm{~mm}$ et $+20 \mathrm{~mm}$, d'autre part les pentes $\mathrm{d} r / \mathrm{d} z$ en chacun de ces points. La source d'ions supposée ponctuelle est située à l'abscisse $z_{0}=-150 \mathrm{~mm}$. Nous avons pris comme conditions initiales les diverses positions des trajectoires au niveau de l'abscisse $(-20 \mathrm{~mm})$ début de la zone active de la lentille, soient :

$$
r_{01}, r_{01}^{\prime}=\frac{r_{01}}{\left|z_{0}-20\right|}=\frac{r_{01}}{130} ; \quad r_{02}, r_{02}^{\prime}=\frac{r_{02}}{130} \ldots
$$

Pour $z<-20$ et $z>+20$, nous avons considéré que les trajectoires étaient rectilignes, en particulier pour déterminer les images $\mathrm{S}_{1}^{\prime}$ et $\mathrm{S}_{2}^{\prime} \ldots$ (cette hypothèse est justifiée par le résultat des calculs, la variation relative de la pente $\mathrm{d} r / \mathrm{d} z$ étant inférieure à $10^{-3}$ entre les abscisses -20 et -17 d'une part, +17 et +20 d'autre part).

b) Résultats. - Un réseau de courbes a été calculé donnant l'abscisse $Z_{\mathrm{S}^{\prime}}$ de l'image de $\mathrm{S}$ en fonction de la tension accélératrice $V_{0}$ des ions, l'ordonnée $r_{0}$ à l'abscisse $-20 \mathrm{~mm}$ étant prise comme paramètre; l'ensemble de ces résultats est résumé sur la figure $5 \mathrm{a}$. L'analyse de ce réseau fait ressortir une divergence des différentes courbes quand la tension accélératrice $V_{0}$ des ions augmente, c'est-à-dire que les ions rapides sont plus influencés que les ions lents

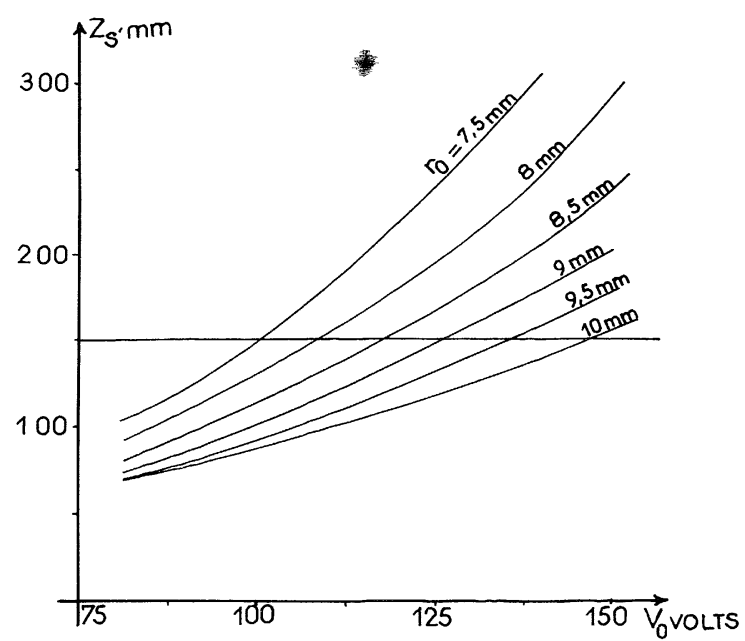

FIG. 5 a. - Courbes donnant $Z_{\mathrm{S}^{\prime}}$ en fonction de $V_{\mathbf{0}}$ pour différentes valeurs de l'ordonnée $v_{0}$ (correspondant à l'abscisse - $20 \mathrm{~mm}$ ).

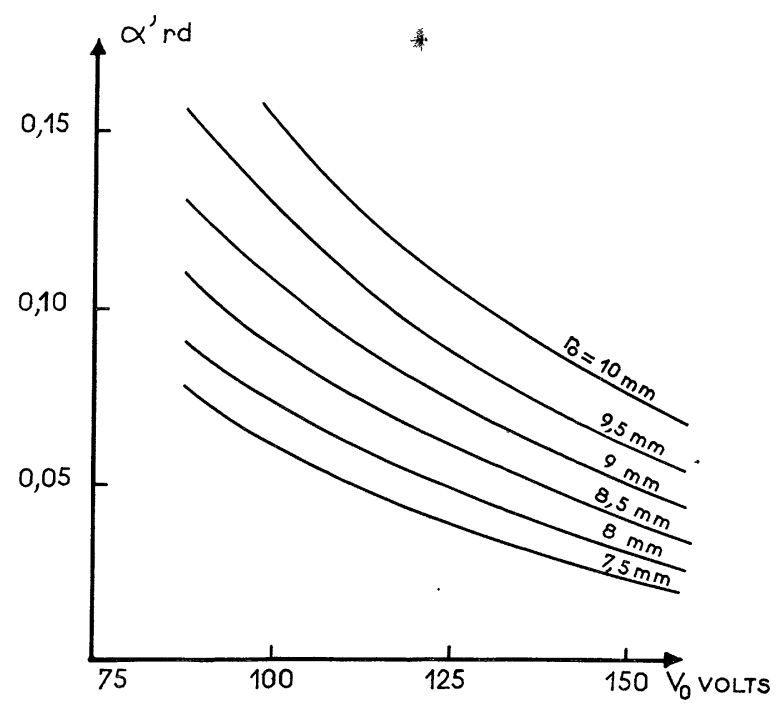

FIG. 5 b. - Courbes donnant $\alpha^{\prime}$ en fonction de $V_{0}$ pour différentes valeurs de l'ordonnée $v_{0}$ (correspondant à l'abscisse - $20 \mathrm{~mm}$ ).

par leur ordonnée initiale. D'autre part, la figure $5 \mathrm{~b}$ donne les variations de la pente $\alpha^{\prime}$ des trajectoires émergentes en fonction des mêmes paramètres que pour la figure 5 a.

V. Vérification expérimentale. - Un montage expérimental reproduisant exactement le schéma théorique ci-dessus a été réalisé ( fig. 6). Un ensemble de pompage secondaire avec piège à azote liquide permet de maintenir un vide de $3 \times 10^{-7}$ torr dans l'enceinte. La source d'ions est une source de type Nier modifiée par Chantreau [2] dont les fentes d'extraction des ions 


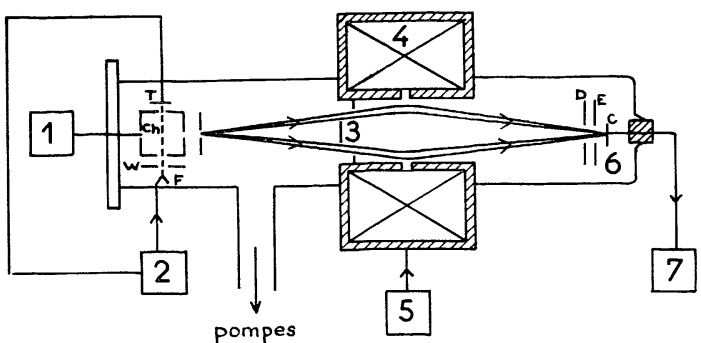

FIG. 6. - Montage expérimental : 1, Dispositif d'introduction du gaz ; 2 , Alimentation de filament régulée par courant de trappe; 3, Diaphragme annulaire; 4, Lentille magnétique; 5 , Alimentation de lentille régulée en courant; 6 , Ensemble collecteur; 7, Électromètre.

ont été remplacées par des orifices circulaires de $1,8 \mathrm{~mm}$ de diamètre. L'orifice de sortie de la source est à l'abscisse $-150 \mathrm{~mm}$. L'émission électronique est régulée par l'intermédiaire du courant de trappe T contrôlant le courant de chauffage du filament F. Un réglage judicieux des tensions du whenelt $\mathrm{W}$ et de la chambre Ch permet d'obtenir un pinceau électronique sensiblement afocal et de faible épaisseur sans avoir à employer un champ magnétique auxiliaire de source. L'hélium préalablement détendu dans un ballon sous vide primaire est introduit dans la source par l'intermédiaire d'une fuite réglable du type « Veeco VL ».

Un dispositif mécanique équivalent à un diaphragme annulaire permet, tout en assurant un bon vide dans l'appareil, de réaliser une nappe conique d'ions de faible épaisseur, traversant le plan d'abscisse $-20 \mathrm{~mm}$ à des ordonnées $r_{0}$ variables; l'épaisseur de la nappe au niveau de ce plan, vis-à-vis d'une source ponctuelle, peut être ainsi réduite à $0,2 \mathrm{~mm}$.

Le courant ionique après passage à travers l'orifice de sortie $\mathrm{D}$, de diamètre $1,8 \mathrm{~mm}$, est reçu par un collecteur $\mathrm{G}$ relié à un amplificateur électrométrique; une électrode intermédiaire $\mathrm{E}$, portée à un potentiel négatif d'environ 30 volts par rapport au collecteur, repousse les électrons secondaires émis par ce dernier. Le déplacement de l'ensemble collecteur présentant des difficultés mécaniques, le diaphragme $\mathrm{D}$ reste fixé à l'abscisse $+150 \mathrm{~mm}$. Pour focaliser les ions à cette distance, on joue donc sur la tension accélératrice $V_{0}$ des ions, le courant magnétisant restant fixé à 1 ampère.

Pour une ouverture donnée du faisceau, on réalise un balayage de la tension accélératrice $V_{0}$ : il lui correspond une courbe de courant ionique ayant l'aspect d'un pic ( fig. 7). L'épaisseur de la nappe ionique étant très faible $(0,2 \mathrm{~mm})$, nous pouvons attribuer sans grand risque d'erreur le sommet du pic de courant ionique, aux ions passant au milieu de cette fente annulaire. Par exemple, pour un diaphragme laissant passer les ions entre les ordonnées 7,4 et $7,6 \mathrm{~mm}$ au niveau de l'abscisse $-20 \mathrm{~mm}$, la tension $V_{0}$ corres-

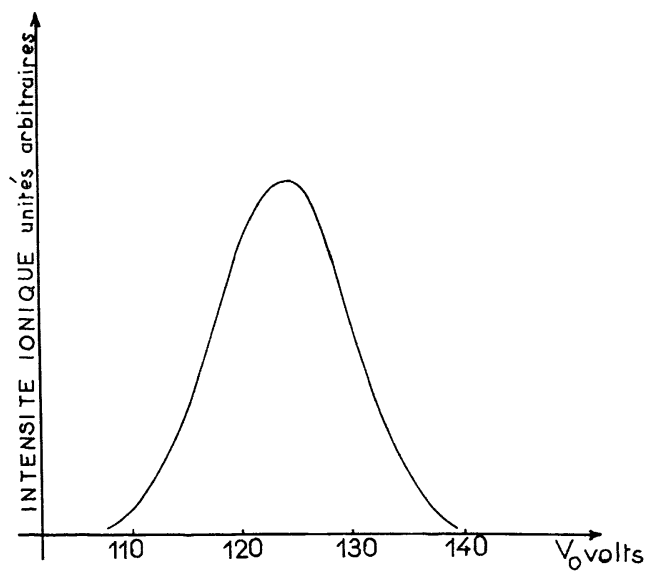

FIG. 7. - Allure du courant ionique en fonction de la tension accélératrice des ions $V_{0}$.

pondant au sommet du pic de courant ionique sera associée à l'ordonnée $r_{0}=7,5 \mathrm{~mm}$.

Nous pouvons ainsi dresser un tableau des tensions accélératrices $V_{0}$, nécessaires pour focaliser les ions d'hélium à l'abscisse $z=+150 \mathrm{~mm}$ en fonction de leur ordonnée initiale $r_{0}$ au niveau de l'abscisse $z=-20 \mathrm{~mm}$. Les résultats sont résumés dans le tableau ci-dessous, les valeurs théoriques étant simplement déduites du réseau de la figure $5 \mathrm{a}$ en traçant sur le graphique une droite d'ordonnée $Z_{\mathrm{s}^{\prime}}=+150 \mathrm{~mm}$ et en déterminant les valeurs de $V_{0}$ correspondant à chaque position de $r_{0}$. Les tensions réelles ont été mesurées à l'aide d'un voltmètre numérique.

TABLEAU I

ORDONNÉE

$\begin{array}{cccc}\begin{array}{c}\text { INITIALE } \\ (\mathrm{mm})\end{array} & \begin{array}{c}V_{0} \text { THÉORIQUE } \\ \text { (volts) }\end{array} & \begin{array}{c}V_{\mathbf{0}} \text { EXPÉR. } \\ \text { (volts) }\end{array} & \begin{array}{c}\text { ÉCART } \Delta V \\ \text { (volts) }\end{array} \\ - & - & - & - \\ 7,5 & 101,5 & 103 & +1,5 \\ 8 & 109 & 110,5 & +1,5 \\ 8,5 & 117,8 & 118,9 & +1,1 \\ 9 & 126 & 126,5 & +0,5 \\ 9,5 & 135,8 & 135 & -0,8 \\ 10 & 146,5 & 144,6 & -1,9\end{array}$

L'analyse du tableau I montre un écart maximum inférieur à $1,5 \%$ entre les valeurs calculées et le; valeurs réelles des tensions accélératrices, écart qui se retrouve entre les distances focales théoriques et expérimentales. Il existe cependant une petite variation systématique, inférieure en valeur relative à l'erreur expérimentale.

Conclusion. - Cette méthode de détermination des trajectoires transgaussiennes apparaît plus précise que les méthodes classiques utilisées jusqu'à pré- 
sent, basées essentiellement sur des procédés mathématiques de détermination du potentiel-vecteur.

Goddard et Klemperer [3] ou Cosslett [4] pour des ouvertures du même ordre de grandeur ont obtenu des écarts entre les valeurs théoriques et expérimentales des éléments géométriques de trajectoires de l'ordre de $5 \%$. D'autre part, s'appuyant sur une grandeur directement accessible à l'expérience, la présente méthode sera particulièrement intéressante dans le cas de lentilles fortement blindées, pour la détermination des trajectoires passant au voisinage de l'entrefer. A première vue, son inconvénient apparaît dans la nécessité de réaliser une série de bobines exploratrices; mais celles-ci ne possédant que quelques spires (ou même une seule dans le cas d'un canal axial de lentille de grand diamètre), la réalisation de ces bobines est en fait très simple et rapide.

Je remercie le personnel du Laboratoire de Calcul numérique de la Faculté des Sciences de Poitiers pour son aide efficace lors des calculs effectués sur IBM 1620.

Je tiens à témoigner ici ma reconnaissance à $\mathrm{M}$. le Professeur R. Vauthier qui m'a proposé cette étude dans son Laboratoire et qui m'a guidé dans son exécution.

\section{BIBLIOGRAPHIE}

[1] Del'homme (M.) et Vauthier (R.), C. R. Acad. Sci. Paris, 1969, 268 B, 219.

[2] Chantreau (J.), C. R. Acad. Sci. Paris, 1967, 265 B, 176.
[3] Goddard (L. S.) et Klempereer (O.), Proc. Phys. Soc., 1944, 56, 378.

[4] CossheiTT, Electron Optics, Clarendon Press, 1950, 100. 